\title{
Neoplastic Fixation to the Prevertebral Compartment by Squamous Cell Carcinoma of the Head and Neck
}

\author{
Laurie A. Loevner ${ }^{1}$ \\ Ingrid L. Ott ${ }^{1}$ \\ David M. Yousem 1,2 \\ Kathleen T. Montone ${ }^{3}$ \\ Erica R. Thaler ${ }^{2}$ \\ Ara A. Chalian ${ }^{2}$ \\ Gregory S. Weinstein ${ }^{2}$ \\ Randal S. Weber ${ }^{2}$
}

Received September 9, 1997; accepted after revision November 6, 1997.

Supported by the Radiological Society of North America Research and Education Fund, Scholar Award Grant.

'Department of Radiology, University of Pennsylvania Medical Center, 3400 Spruce St., Philadelphia, PA 19104. Address correspondence to L. A. Loevner.

${ }^{2}$ Department of Otorhinolaryngology; Head and Neck Surgery, University of Pennsylvania Medical Center, Philadelphia, PA 19104

${ }^{3}$ Department of Pathology, University of Pennsylvania Medical Center, Philadelphia, PA 19104.

AJR 1998;170:1389-1394

0361-803X/98/1705-1389

(C) American Roentgen Ray Society

OBJECTIVE. The purpose of this study was to determine the accuracy of MR imaging in determining fixation of squamous cell carcinomas to the prevertebral space.

MATERIALS AND METHODS. MR images of 15 patients with large pharyngeal carcinoma $(n=13)$ or laryngeal carcinomas with pharyngeal extension $(n=2)$ were retrospectively reviewed independently by two head and neck radiologists who were unaware of the surgical findings. MR images were evaluated for four criteria in the prevertebral longus muscle complex: muscle concavity, irregular tumor-muscle interface, T2 hyperintensity, and enhancement. All patients underwent panendoscopy where fixation or mobility of the tumor relative to the prevertebral fascia was assessed by manual manipulation. Tumors in six patients were fixed to the prevertebral space and inoperable. In nine patients whose tumors were not fixed, open neck explorations were performed and tumors were resected in seven patients. MR findings were compared with panendoscopy in all patients and with intraoperative assessment in nine patients.

RESULTS. Eleven of 15 patients had at least two of the MR imaging criteria present. None of the MR findings were both sensitive and specific for tumor fixation. Although muscle concavity and enhancement each had a sensitivity of $88 \%$, both criteria suffered from low specificity (14\% and $29 \%$, respectively). An irregular tumor-muscle interface and muscle T2 hyperintensity were criteria that suffered from both low sensitivity and specificity. Accuracy of the imaging criteria independently ranged from $53 \%$ to $60 \%$.

CONCLUSION. Although abnormal muscle contour, T2 hyperintensity, and enhancement are frequently present in neck carcinomas that are fixed to the prevertebral space, these findings may also be present in patients in whom the tumor is mobile and resectable. MR imaging may not be able to differentiate between neoplastic fixation and nonneoplastic changes in the prevertebral space.

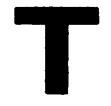

he treatment of squamous cell carcinoma of the head and neck is complex. The need for and type of surgery are guided by the site of origin of the primary tumor and stage of disease at the time of clinical presentation. Staging is determined by many factors, including tumor size and origin, submucosal spread into the deep spaces of the neck, cartilage invasion, lymphadenopathy, and distant metastases. Direct visualization combined with endoscopy is sensitive in evaluating the mucosa of the aerodigestive tract [1-3]. However, even extensive neoplastic infiltration of the deep spaces surrounding the upper aerodigestive tract is often difficult to detect on physical and endoscopic examination. As a result, disease is frequently understaged clinically [4].
Radiologic imaging has played an increasingly important role in the staging of head and neck cancer, including the detection of clinically occult lymphadenopathy, cartilage invasion, and tumor spread into the submucosal spaces of the neck [5-12]. However, investigation into the ability of imaging to predict the presence of tumor spread to the prevertebral compartment has been scant. Neoplastic spread directly into the prevertebral space or fixation of tumor to the prevertebral fascia is significant in that it usually renders the tumor inoperable and often incurable [13]. Presently, neoplastic fixation and extension to the prevertebral space may only be determined intraoperatively by manual manipulation under general anesthesia. Fixed tumors are deemed inoperable and surgery is 
aborted. Therefore, it would be extremely valuable to be able to reliably determine the presence or absence of prevertebral fixation by preoperative imaging.

The purpose of this study was to determine the usefulness of MR imaging in detecting neoplastic spread to the prevertebral space in patients with advanced oropharyngeal, hypopharyngeal, and laryngeal cancer. To do this, MR imaging findings were compared with observations during panendoscopy and open neck exploration.

\section{Materials and Methods}

The MR images of 57 patients with primary pharyngeal or laryngeal cancer seen in a 3-year perod (1993-1996) in the head and neck cancer center at our institution were retrospectively reviewed. The MR images were evaluated for preservation of the prevertebral fat between the tumor and the prevertebral musculature (longus colli-longus capitis complex). The retropharyngeal fat plane is readily identified on unenhanced T1-weighted MR images because of its hyperintensity relative to surrounding tissues in the neck (muscle and lymphoid tissue) (Fig. 1). This fat plane at the tumor-muscle interface was obliterated in 20 patients; however, five patients were eliminated from the study because no clinical or intraoperative assessment was available. The MR images of the remaining $15 \mathrm{pa}$ tients (nine men, six women), who had a mean age of 62 years (range, 48-78 years), were selected for inclusion in this study. All patients had squamous cell carcinoma with the site of origin in the oropharynx $(n=8)$, the hypopharynx $(n=5)$, and
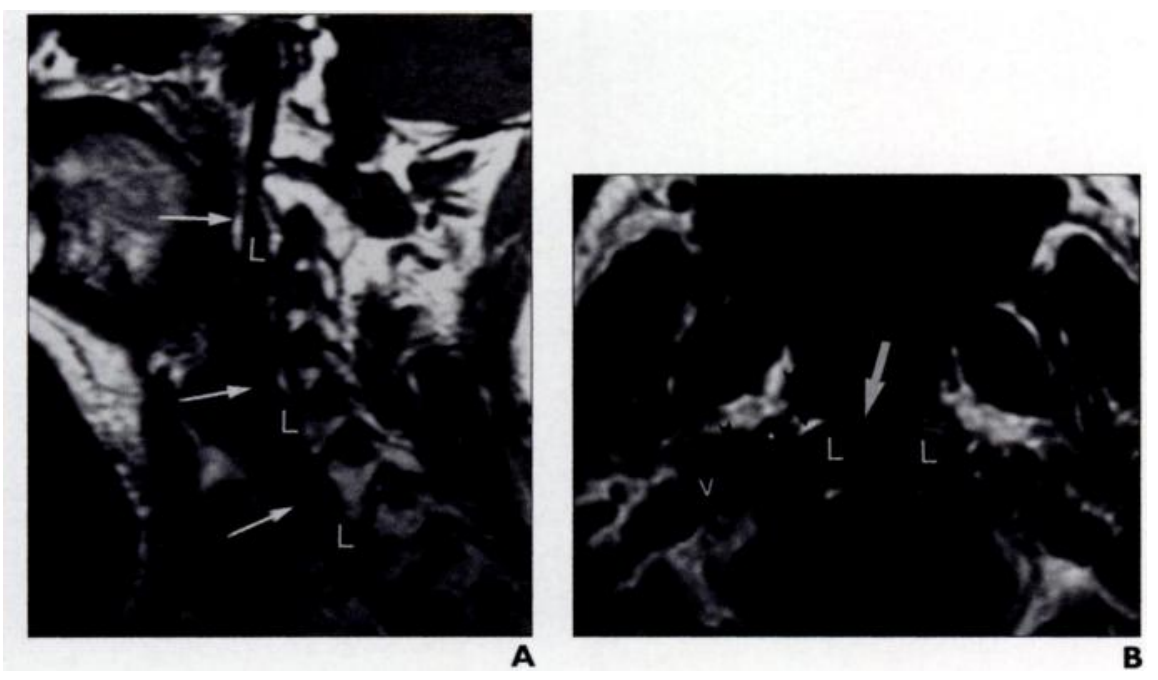

Fig. 1.- Normal anatomy in 46 -year-old man.

A. Off midline sagittal T1-weighted MR image (600/17 [TR/TE]; section thickness, $5 \mathrm{~mm}$ ) shows prevertebral compartment of perivertebral space demarcated by precervical retropharyngeal fat seen as hyperintense linear band (arrows). Note longus muscle complex (L).

B, Axial T1-weighted MR image (600/17; section thickness, $5 \mathrm{~mm}$ ) obtained at level of oropharynx shows prevertebral compartment demarcated by precervical retropharyngeal fat (arrows). Note longus muscle complex (L) internal carotid artery (asterisk), and jugular vein (v).

the larynx with extension to the pharynx $(n=2)$. All patients underwent panendoscopy under general anesthesia to assess for the presence or absence of tumor fixation to the prevertebral compartment. Patients thought to be surgical candidates during panendoscopy underwent open neck exploration and surgical resection.

All examinations were performed with a Signa 1.5-T scanner (General Electric Medical Systems, Milwaukee, WI) using an anterior-posterior volume neck coil (Medical Advances, Madison, WI). The MR imaging protocol consisted of standard spin-echo sagittal T1-weighted images (TR/TE range, 600/11-17) followed by fat-suppressed axial fast spin-echo T2-weighted (TR range/TE, $3500-4000 / 90)$ and axial spin-echo T1-weighted images (600/11-17). Images of the neck extended from the level of the cavernous sinus to the thoracic inlet. After the administration of $0.1 \mathrm{mmol}$ kg gadopentetate dimeglumine IV (Magnevist; Schering, Berlin, Germany) axial spin-echo T1weighted imaging was repeated using the same parameters as the unenhanced scans. Fast spin-echo and contrast-enhanced studies were obtained with the application of frequency-selective fat-saturation techniques. For the sagittal $\mathrm{T} 1$-weighted localizing MR images, a slice thickness of $5 \mathrm{~mm}$ with a gap of $1 \mathrm{~mm}$ and a 30-cm field of view were used. For all other sequences, a slice thickness of 5 $\mathrm{mm}$ with interleaved images was used. Other imaging parameters included one or two excitations, 24 to $26-\mathrm{cm}$ field of view, a $256 \times 128$ matrix for sagittal and contrast-enhanced T1-weighted images, and a $256 \times 192$ matrix for axial unenhanced T1- and T2-weighted images.

Retrospective image analysis was performed independently by two head and neck radiologists

.

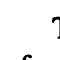
criteria assessed for a total of 60 interpretations. The observers agreed in $\mathbf{5 7}$ of 60 readings $(\kappa=.88)$ and resolved the three discrepant interpretations by consensus. The individual criteria for prevertebral fixation (ipsilateral muscle concavity, irregular muscular 
MR Imaging of Squamous Cell Carcinoma of the Head and Neck

\begin{tabular}{|c|c|c|c|c|}
\hline \multirow{2}{*}{\begin{tabular}{|l|} 
TABLE I \\
MR Findings
\end{tabular}} & \multicolumn{4}{|c|}{$\begin{array}{l}\text { Findings Revealed by MR } \\
\text { Imaging in } 15 \text { Patients }\end{array}$} \\
\hline & $\begin{array}{l}\text { True- } \\
\text { Positive }\end{array}$ & $\begin{array}{l}\text { False- } \\
\text { Positive }\end{array}$ & \begin{tabular}{|c|} 
True- \\
Negative
\end{tabular} & $\begin{array}{l}\text { False- } \\
\text { Negative }\end{array}$ \\
\hline $\begin{array}{r}\text { Concavity } \\
(n=13)\end{array}$ & 7 & 6 & 1 & 1 \\
\hline $\begin{array}{l}\text { Irregular } \\
\quad \text { borders } \\
(n=9)\end{array}$ & 5 & 4 & 3 & 3 \\
\hline $\begin{array}{c}\text { T2 hyperin- } \\
\text { tensity } \\
(n=8)\end{array}$ & 4 & 4 & 3 & 4 \\
\hline $\begin{array}{l}\text { Enhanc3- } \\
\qquad \begin{array}{l}\text { ment } \\
(n=12)\end{array}\end{array}$ & 7 & 5 & 2 & 1 \\
\hline
\end{tabular}

border at the tumor-muscle interface, muscle T2 hyperintensity, and muscle enhancement) suffered from both low specificity (range. 14

\begin{tabular}{|c|c|c|c|c|c|c|}
\hline TABLE 2 & \multicolumn{6}{|c|}{$\begin{array}{l}\text { Prevertebral Muscle Fixation: Comparison of Criteria Revealed by MR } \\
\text { Imaging in } 15 \text { Patients }\end{array}$} \\
\hline Criter & eria & $\begin{array}{l}\text { Sensitivity } \\
(\%)\end{array}$ & $\begin{array}{l}\text { Specificity } \\
(\%)\end{array}$ & $\operatorname{PPV}(\%)$ & NPV (\%) & Accuracy \\
\hline Concavity & & 88 & 14 & 54 & 50 & 53 \\
\hline Irregular border & & 62 & 43 & 56 & 50 & 53 \\
\hline T2 hyperintensit & & 50 & 43 & 50 & 43 & 47 \\
\hline Enhancement & & 88 & 29 & 58 & 67 & 60 \\
\hline
\end{tabular}

Note. - PPV = positive predictive value, NPV = negative predictive value .

$43 \%$ ) and low accuracy (range, 53-60\%) due to a high false-positive rate (Tables 1 and 2). Although the presence of muscle concavity and enhancement each showed $88 \%$ sensitivity for prevertebral fixation. high false-positive rates resulted in low specificities $(14 \%$ and $29 \%$, respectively). No correlation existed between prevertebral muscle fixation and the

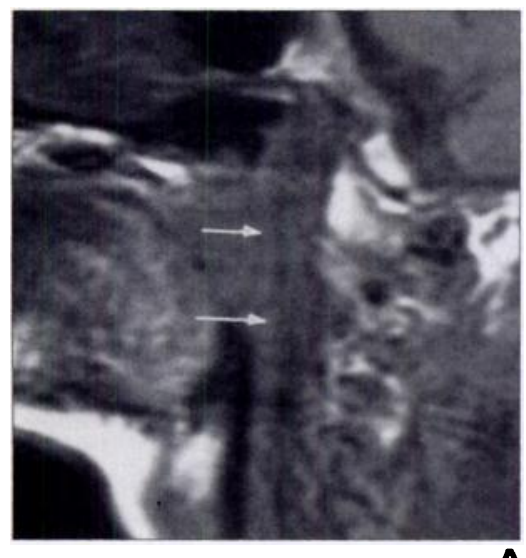

A
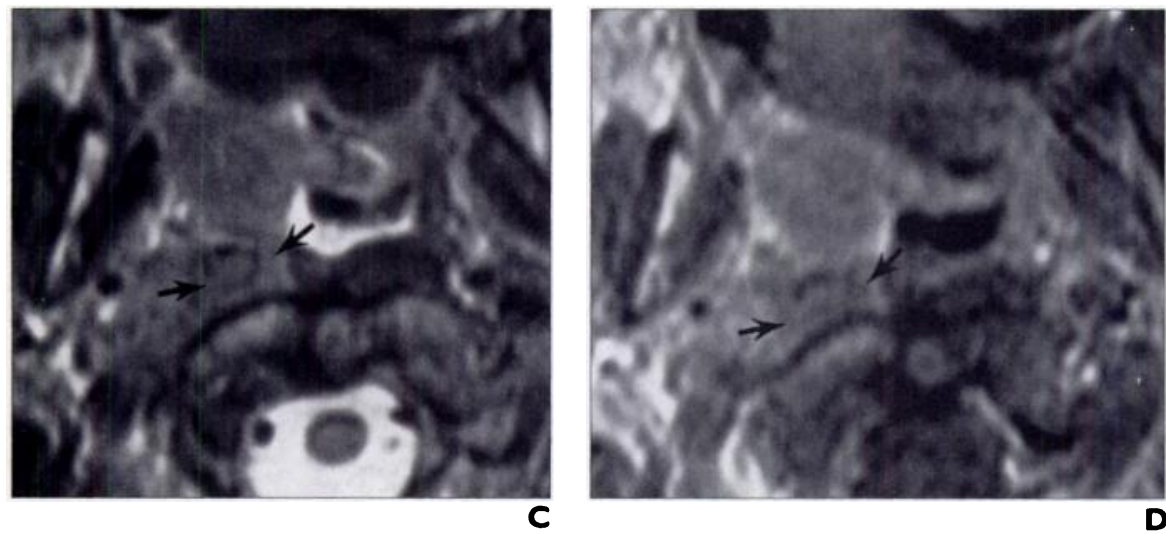

Fig. 2. - 49-year-old woman with primary carcinoma of oropharynx.

A, Sagittal T1-weighted MR image (600/17 [TR/TE]; section thickness, $5 \mathrm{~mm}$ ) shows attenuation of right retropharyngeal fat plane (arrows).

B, Axial T1-weighted MR image (600/17; section thickness, $5 \mathrm{~mm}$ ) shows attenuation of right retropharyngeal fat plane (arrows) and mild enlargement of right longus muscle complex; compare with normal left side (L). Note retropharyngeal nodal mass (N).

C, Axial T2-weighted MR image (3500/90; section thickness, $5 \mathrm{~mm}$ ) reveals abnormally high signal intensity in longus muscle complex (arrows)

D, Contrast-enhanced axial T1-weighted MR image (600/17; section thickness, $5 \mathrm{~mm}$ ) shows enhancement of prevertebral muscle (arrows). On intraoperative panendoscopy, tumor was found to be fixed to prevertebral musculature, making resection impossible. presence or absence of either irregular muscular borders at the tumor-muscle interface or T2 hyperintensity (Figs. 2 and 3). The presence of enhancement of the muscle was the most likely predictor of tumor fixation (positive predictive value, $58 \%$ ) and was the most accurate criterion at just $60 \%$. The correlation between the number of positive MR criteria and the presence of fixation was poor. Some patients with few positive criteria had fixation. Even in the seven patients with all four criteria present, only three had tumors that had prevertebral fixation on panendoscopy.

Although the sample size of patients was too small to apply a grading system for each of the MR criteria, we did note that in patients with fixation of tumor to the prevertebral compartment the degree of $\mathrm{T} 2$ hyperintensity and enhancement within the prevertebral muscle was usually more prominent than in those patients without fixation.

\section{Discussion}

The prevertebral space is the anterior compartment of the perivertebral space. It is located behind the retropharynx. invested anteriorly by a layer of fat and the deep cervical fascia. The prevertebral space. encapsulated by the deep layer of the deep cervical fascia. contains a fat plane that is located between the two layers of the precervical fascia, the anterior prevertebral muscles (longus capitis, longus colli, rectus capitis anterior, and rectus capitis lateralis), the paraspinal musculature, and the vertebral bodies $[14,15]$ (Fig. 1). The musculus longus colli runs along the ventral surface of the vertebral bodies, extending from the atlas to the third or fourth vertebral body. The musculus longus capitis arises from the base of the occipital bone and extends to the anterior tubercles of the transverse processes of the third through sixth vertebrae. The longus colli and capitis muscles are the most vulnerable of the prevertebral muscles to the spread of disease processes by virtue of their proximity and contiguity to the pharyn- 

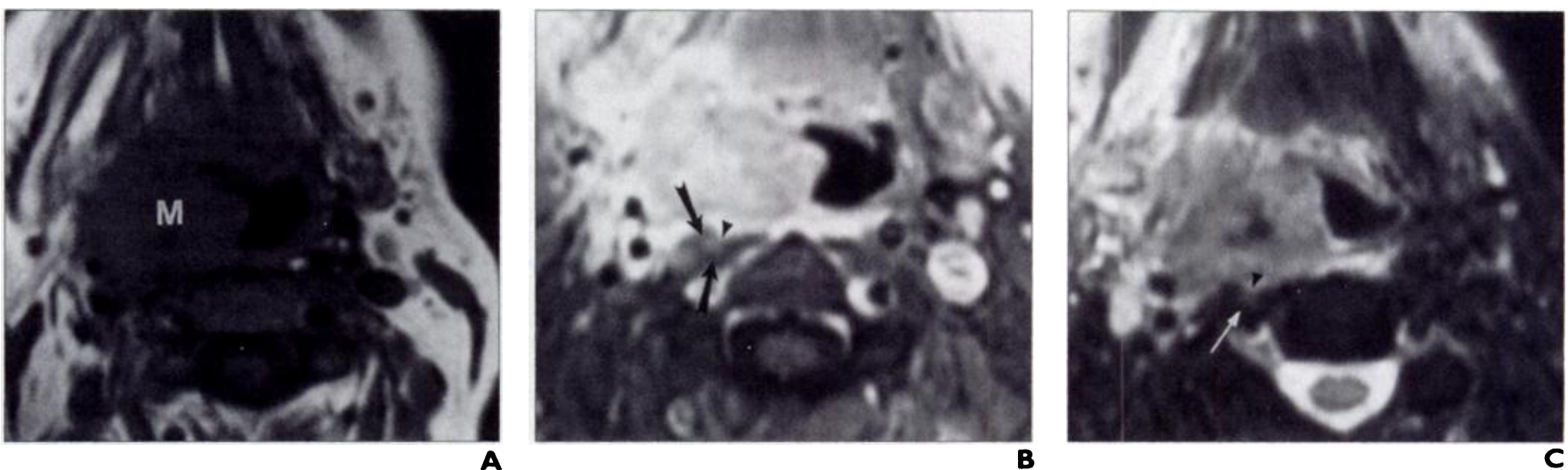

Fig. 3. - 59-year-old woman with cancer of right oropharynx. Prevertebral invasion was suspected on basis of MR findings; however, intraoperatively, mass was not fixed to prevertebral structures and tumor was resected. Final histology revealed no evidence of tumor in surgical margins.

A, Axial T1-weighted MR image (600/17 [TR/TE]; section thickness, $5 \mathrm{~mm}$ ) shows large oropharyngeal mass (M) with obliteration of fat between mass and prevertebral muscle. B, Axial contrast-enhanced T1-weighted MR image (600/17; section thickness, $5 \mathrm{~mm}$ ) reveals concavity of longus muscle complex (arrowhead) and muscle enhancement (arrows).

C, Axial T2-weighted MR image (3500/90; section thickness, $5 \mathrm{~mm}$ ) shows concavity of prevertebral muscle complex (arrowhead) and abnormal signal intensity (arrow).

geal mucosal and retropharyngeal spaces, respectively $[15,16]$. Although the deep cervical fascia is a relatively resilient barrier to the extension of disease processes from the pharyngeal mucosal and the deep nonmucosal spaces of the anterior neck, spread of disease including infectious and neoplastic processes may occur [15-17].

Spread of tumor to the prevertebral compartment in the form of frank neoplastic invasion of the prevertebral musculature may occur. However, prevertebral extension may also be manifested by fixation of tumor to the prevertebral fascia without violation of the fascia or by tumor penetration through this fascia but without direct extension into the muscle. In head and neck cancer, any form of prevertebral extension (including only fixation to the prevertebral fascia) is significant in that it often renders the tumor inoperable and the patient incurable because surgeons are unable to obtain tumor-free surgical margins. The prevertebral musculature is intimately associated with and interdigitates with the fascia of the prevertebral column. Hence, resection of the prevertebral muscle is difficult, dangerous, and unlikely to achieve complete resection of the gross tumor. In addition, partial surgical debulking of macroscopic tumor is associated with a poor clinical and functional outcome.

In patients with squamous cell carcinoma of the pharynx at risk for prevertebral space invasion who are otherwise surgical candidates (no encasement of the internal carotid artery and no intracranial perineural extension through the skull base foramina), determination of tu- mor fixation to the prevertebral compartment is presently made by digital manipulation on panendoscopy under general anesthesia. If unequivocal fixation of the tumor exists, open exploration is often not necessary. Otherwise. open neck exploration is usually performed through the side of the neck not involved by the primary tumor. If both sides of the neck appear to be involved by tumor, the side with less disease is used for access. The technique involves retraction of the carotid sheath and its contents laterally and rotation of the larynx medially. Then a finger is run superiorly to inferiorly in the plane anterior to the prevertebral muscle to determine if the tumor is fixed. If the tumor appears adherent to the deep cervical fascia. but not through it, some head and neck oncology surgeons will resect the fascia with the tumor. In some cases, a biopsy of regions of suspicion in the muscle may be performed. and the sample is evaluated by frozen section to determine if the muscle has been invaded by tumor [13]. If tumor extension through the prevertebral fascia to the muscle is found. surgery is aborted and patients are treated with radiation therapy or combined radiation and chemotherapy. If no direct evidence of tumor extension exists, extirpative surgery is performed. Although the possibility of microinvasion cannot be excluded until the final histology is reported. this is of less concern to the surgeon because most patients are treated with radiation therapy after surgery.

Imaging plays a critical role in the staging of head and neck cancer including detection of lymphadenopathy, cartilage invasion. and tu- mor spread into the submucosal spaces of the neck [5-12, 18-22]. The goal of the head and neck radiologist is not only to help direct the appropriate surgery for a patient with cancer but also to determine which tumors are surgically unresectable. Assessment of the prevertebral space is vital in this regard: however. little radiologic investigation has been done to determine if imaging findings exist that would indicate the presence or absence of neoplastic extension to the prevertebral compartment. In a study by Righi et al. [13]. CT scans in 29 patients with $\mathrm{T} 3$ and $\mathrm{T} 4$ pharyngeal carcinomas were retrospectively reviewed by two neuroradiologists and compared with operative findings during open neck exploration. In 17 patients, the CT scans were interpreted as negative for neoplastic spread to the prevertebral muscles using the criterion of preservation of the fat plane between the tumor and the muscle. Intraoperatively. three of these 17 patients were found to have prevertebral invasion and surgery was aborted. Hence, maintenance of the precervical fat plane correctly predicted absence of prevertebral involvement $82 \%$ of the time. The preoperative $C T$ scans of 12 patients were interpreted as suggestive of prevertebral invasion on the basis of the obliteration of the fat plane between the tumor and the prevertebral muscle and asymmetric enlargement of the muscle complex contiguous with the tumor. Three of these patients $(25 \%)$ had involvement of the prevertebral space on intraoperative assessment. The remaining nine patients in this group underwent resection, and, in three, microscopic tumor along the deep margin of the surgical 


\section{MR Imaging of Squamous Cell Carcinoma of the Head and Neck}
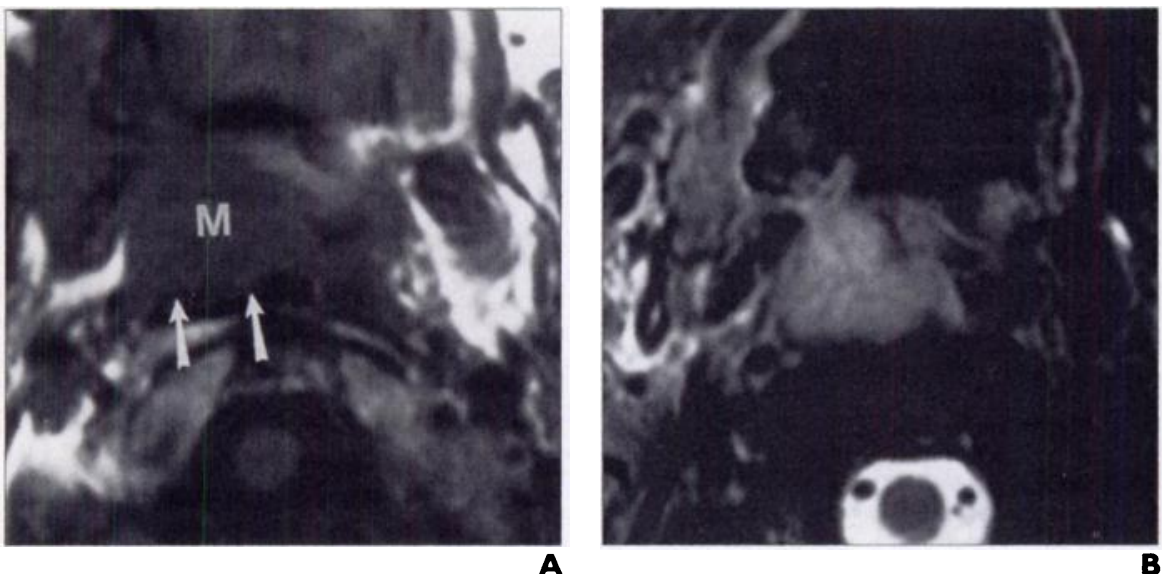

Fig. 4. - 52-year-old man with cancer of right oropharynx (tumor also involves right superior alveolus and retromolar trigone).

A, Axial T1-weighted MR image (600/17 [TR/TE]; section thickness, $5 \mathrm{~mm}$ ) shows large oropharyngeal mass (M) compressing prevertebral compartment with concavity of muscle complex and irregular tumor-muscle interface (arrows).

B. Axial T2-weighted MR image (3500/85; section thickness, $5 \mathrm{~mm}$ ) acquired at same axial level as A shows similar findings along prevertebral compartment. No signal intensity abnormality is seen in longus muscles. Tumor was freely mobile during surgery with tumor-free margins on histologic examination of resected specimen.

specimen was present on final histology: however, the prevertebral muscle fascia was intact and uninvolved on direct inspection. Overall. this study suggests that CT suffers from low sensitivity and low specificity in revealing prevertebral space invasion.

In the present study. MR findings were correlated with intraoperative assessment in 15 patients with large pharyngeal or laryngeal primary tumors that obliterated the prevertebral fat such that the tumors appeared contiguous with the prevertebral muscles (Figs. 4 and 5). The study by Righi et al. [13] suggested that maintenance of the precervical fat plane on CT correctly predicted absence of macroscopic prevertebral involvement $82 \%$ of the time. With the improved soft-tissue resolution of MR imaging and its multiplanar capabilities. the accuracy in predicting preservation of the precervical fat could potentially be higher. Nonetheless, the surgeons at our institution believe that preservation of this fat plane on imaging in the absence of other indicators of inoperability warrants evaluation by panendoscopy or surgical exploration even in the event that occasionally a tumor may be found to be unresectable during surgery.

In this study, MR images were analyzed for the presence or absence of ipsilateral muscle concavity. irregular tumor-muscle interface. abnormal $\mathrm{T} 2$ hyperintensity in the muscle. and muscle enhancement. At least two of these imaging findings were present in 11 of the $15 \mathrm{pa}$ tients, and none of these findings individually or together were consistently reliable in distinguishing those patients with prevertebral ex- tension (eight) from those without (seven) (Tables 1 and 2). Although the sample size of patients was too small to apply a grading system for each of the MR criteria, we did note that in patients with fixation of tumor to the prevertebral compartment the degree of $\mathrm{T} 2$ hyperintensity and enhancement within the prevertebral muscle was usually more prominent than in those patients without fixation. The presence of muscle enhancement was the most sensitive $(88 \%)$ and the most accurate $(60 \%)$ criterion for tumor fixation. but it was not an accurate indicator of neoplastic spread. We speculate that a reactive inflammatory infiltration produced by the squamous cell carcinoma of the neck may account for the muscle $\mathrm{T} 2$ hyperintensity or enhancement. Some basis for this hypothesis can be found in the musculoskeletal literature [23]. Musculoskeletal neoplasms, tumor invading muscle, and nonneoplastic muscle edema may be hyperintense on T2-weighted images due to increased water content and may show enhancement after contrast material administration [23, 24]. A study by Lang et al. [24], who attempted to distinguish peritumoral edema from tumor, suggested that these entities may be differentiated by dynamic scanning and spatial mapping of instantaneous enhancement rates. These authors found that viable tumor showed greater and more rapid increases in $\mathrm{Tl}$ weighted signal intensity after contrast material administration than perineoplastic edema. which showed lower and more gradual increases in signal intensity. The applicability of MR imaging in the head and neck remains to be determined.

In conclusion, using the current MR techniques described herein, MR imaging seems unable to consistently distinguish neoplastic fixation or invasion of the prevertebral musculature from benign reaction. because both

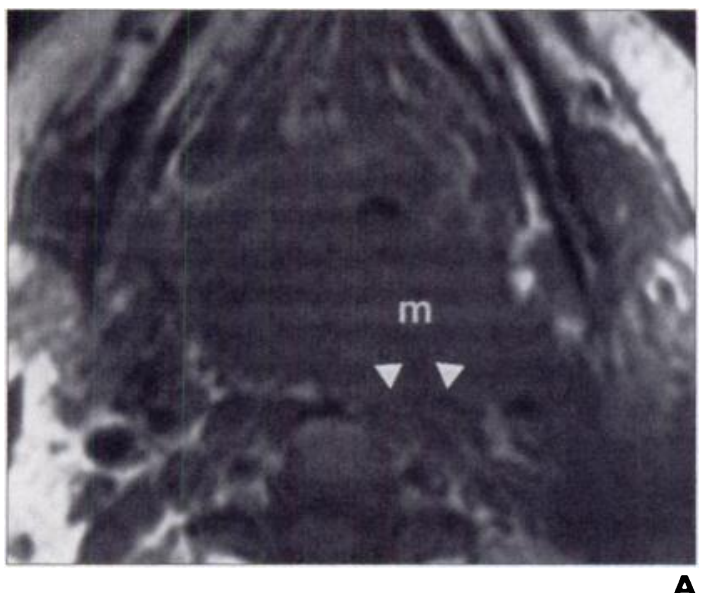

A

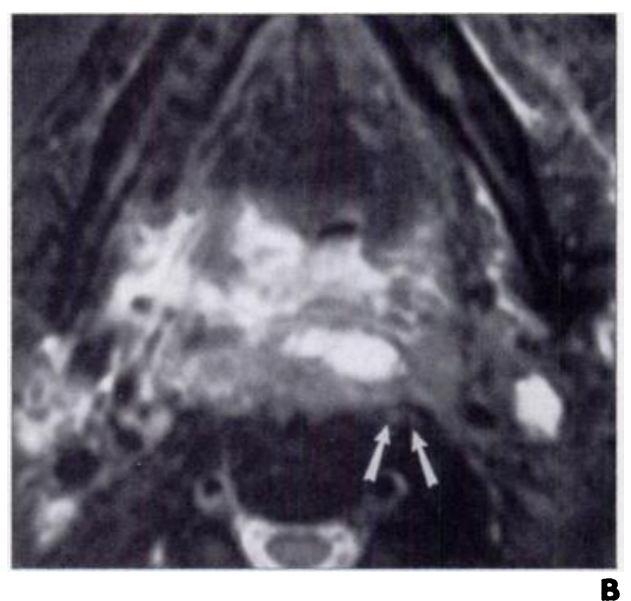

Fig. 5.-52-year-old man with pharyngeal cancer.

A, Axial T1-weighted MR image $600 /$ 17 [TR/TE]; section thickness, $5 \mathrm{~mm}$ ) shows large pharyngeal mass (m) obliterating retropharyngeal fat and mildly compressing prevertebral muscle complex (arrowheads).

B. Axial T2-weighted MR image (3500/85; section thickness, $5 \mathrm{~mm}$ ) shows increased signal intensity in muscle (arrows). On panendoscopy, fixation of mass was equivocal. On open neck exploration tumor was not freely mobile, and intraoperative biopsy revealed tumor invading prevertebral muscle. 
processes can have similar imaging findings. Abnormal T2 signal intensity or enhancement should not be taken as an absolute indication of fixation or invasion because it may be seen in patients whose tumors are resectable, as in this study. Currently, determining fixation or invasion of primary head and neck carcinomas to the prevertebral compartment must be made intraoperatively. The number of patients in our study was small $(n=15)$, and a study with a larger sample size is needed for further evaluation of this observation. Other limitations include an imperfect gold standard for determining prevertebral muscle invasion-namely, lack of pathologic proof of tumors determined to be fixed on panendoscopy. The possibility of microscopic invasion in patients with intact retropharyngeal-precervical fat planes was also not addressed in this study.

\section{References}

1. Freeman DE, Mancuso AA, Parsons JT, Mendenhall WM, Million RR. Irradiation alone for supraglottic larynx carcinoma: can CT findings predict treatment results? Int J Radiat Oncol Biol Phys 1990; 19:485-490

2. Sanghvi V. The new combined surgical approach for cancer involving the base of tongue-supraglottic complex. Lanyngoscope 1994:104:725-730

3. Loveday EJ, Bleach NR, Van Hasselt CA, Metreweli C. Ultrasound imaging in laryngeal cancer: a preliminary study. Clin Radiol 1994;49: 676-682
4. Zeitels SM, Vaughn CW. Preepiglottic space invasion in "early" epiglottic cancer. Ann Otol Rhinol Laryngol 1991:100:789-792

5. Ali YA, Saleh EM, Mancuso AA. Does conventional tomography still have a place in glottic cancer evaluation? Clin Radiol 1992:45:114-119

6. Mafee MF, Schild JA, Valvassori GE, Capek V. Computed tomography of the larynx: correlation with anatomic and pathologic studies in cases of laryngeal carcinoma. Radiology 1983; 147:123-128

7. Hoover LA, Calcaterra TC, Walter GA, Larrson SG. Pre-operative CT scan evaluation for laryngeal carcinoma: correlation with pathologic findings. Lanyngoscope 1984;94:310-315

8. Gregory RT, Lloyd GAS, Michaels L. Computed tomography of the larynx: a clinical and pathologic study. Head Neck Surg 1981;3:284-296

9. Tien RD, Hesselink JR, Chu PK, Szumowski J. Improved detection and delineation of head and neck lesions with fat-suppression spin-echo MR imaging. AJNR 1991;12:19-24

10. van den Brekel MWM, Stel HV, Castelijns JA, et al. Cervical lymph node metastasis: assessment of radiologic criteria. Radiology 1990;177:379-384

11. Castelijns JA, Gerritsen GJ, Kaiser MC, et al. Invasion of laryngeal cartilage by cancer: comparison of CT and MR imaging. Radiology 1987: 166:199-206

12. Yousem DM, Som PM, Hackney DB, Schwaibold F, Hendrix RA. Central nodal necrosis and extracapsular neoplastic spread in cervical lymph nodes: MR imaging versus CT. Radiology 1992; 182:753-759

13. Righi PD, Kelley DJ, Ernst R, et al. Evaluation of prevertebral muscle invasion by squamous cell carcinoma. Arch Otolaryngol Head Neck Surg 1996; 122:660-663

14. Madison MT. Remley KB, Latchaw RE. Mitchell SL. Radiologic diagnosis and staging of head and neck squamous cell carcinoma. Radiol Clin North Am 1994:32:163-181

15. Parker GD, Harnsberger HR. Radiologic evaluation of the normal and diseased posterior cervical space. AJR 1991;157:161-165

16. Davis WL, Harnsberger HR. Smoker WR, et al. Retropharyngeal space: evaluation of normal anatomy and diseases with CT and MR imaging. Radiology 1990: 174:59-64

17. Paonessa DF. Goldstein JC. Anatomy and physiology of head and neck infections (with emphasis on the fascia of the face and neck). Otolarningol Clin North Am 1976:9:561-580

18. Som PM. Lymph nodes of the neck. Radiology 1987:165:593-600

19. Dooms GC. Hricak H. Crooks LE. et al. Magnetic resonance imaging of the lymph nodes: comparison with CT. Radiology 1984;153:719-724

20. Muraki AJ, Mancuso AA, Harnsberger HR. Metastatic cervical adenopathy from tumors of unknown origin: role of CT. Radiology 1984:152: 749-753

21. Dooms GC. Hricak H. Radiologic imaging modalities, including magnetic resonance, for evaluating lymph nodes. West J Med 1986; 144:49-57

22. Becker M, Zbaren P. Laeng H. Stoupis C. Porcellini B. Vock P. Neoplastic invasion of the laryngeal cartilage: comparison of MR imaging and CT with histopathologic correlation. Radiology 1995: 194:661-669

23. Seeger LL, Widoff BE, Bassett LW, Rosen G, Eckardt JJ. Preoperative evaluation of osteosarcoma: value of gadopentetate dimeglumine-enhanced MR imaging. AJR 1991:157:347-351

24. Lang P. Honda G, Roberts T, et al. Musculoskeletal neoplasm: perineoplastic edema versus tumor on dynamic postcontrast MR images with spatial mapping of instantaneous enhancement rates. $R a$ diology 1995; 197:831-839 